\title{
E75-a safe and effective vaccine for the prevention of disease recurrence
}

Breast cancer is the most common malignancy in women and even after curative treatment, approximately 10-20\% of breast cancer survivors will develop disease recurrence. A recent study, published in the Annals of Oncology, reports that administration of the E75 vaccine (nelipepimut-S) in the adjuvant setting is safe and effective in preventing disease recurrence in disease-free patients at high-risk of breast cancer relapse.

E75 is a HER2-derived peptide that can elicit a specific immune response. Mittendorf and colleagues have used this peptide in combination with an immunoadjuvant-the granulocytemacrophage colony-stimulating factor (GM-CSF) — and have conducted phase I/II trials to document the safety, immunogenicity and clinical efficacy of the E75 vaccine.

Of the 187 evaluable patients, 108 were included in the vaccination group and 79 in the untreated control group, on the basis of their HLA-A2/A3 type.
The primary analysis of the combined trials was initiated at 18 months follow-up and provided encouraging results with a recurrence rate of $5.6 \%$ in the vaccination group compared with $14.2 \%$ in the control group. Importantly, the trial follow-up was extended to 60 months and a booster programme was included. The final analysis indicates that toxic effects were minimal, and that the 5-year diseasefree survival was $89.7 \%$ in the vaccination group versus $80.2 \%$ in the control group.

This study highlights the safety and clinical efficacy of the E75 vaccine and, on the basis of these data, a phase III trial has been initiated with the aim of evaluating the optimal dose and booster programme for this vaccine.

\section{Alessia Errico}

Original article Mittendorf, E. A. et al. Final report of the phase $\mathrm{I} / \mathrm{II}$ clinical trial of the $\mathrm{E} 75$ (nelipepimut-S) vaccine with booster inoculations to prevent disease recurrence in high-risk breast cancer patients. Ann. Oncol. doi:10.1093/ annonc/mdu211 